Cahiers de Narratologie

Analyse et théorie narratives

\title{
Le chapitre comme phénomène médiatique dans La Jeunesse de Picsou de Don Rosa
}

Anaïs Goudmand

\section{(2) OpenEdition}

\section{Journals}

\section{Electronic version}

URL: http://journals.openedition.org/narratologie/8736

DOI: 10.4000/narratologie.8736

ISSN: 1765-307X

Publisher

LIRCES

Electronic reference

Anaiis Goudmand, «Le chapitre comme phénomène médiatique dans La Jeunesse de Picsou de Don Rosa », Cahiers de Narratologie [Online], 34 | 2018, Online since 21 December 2018, connection on 25 April 2019. URL : http://journals.openedition.org/narratologie/8736 ; DOI : 10.4000/narratologie.8736

This text was automatically generated on 25 April 2019

Article L.111-1 du Code de la propriété intellectuelle. 


\title{
Le chapitre comme phénomène médiatique dans La Jeunesse de Picsou de Don Rosa
}

\author{
Anaïs Goudmand
}

1 Selon Richard Saint-Gelais, la culture médiatique se caractérise par une «émancipation transfictionnelle du personnage» (2011: 373), qui se détache des récits qui le mettent originellement en scène pour être recyclé à travers diverses productions allographes. Ce phénomène serait "favoris[é] par des facteurs quantitatifs comme la multiplicité des médias, des versions et des “auteurs" (de sorte à la limite qu'aucun n'en est plus un) [...] » (2011: 377). On assiste ainsi à un effacement de la figure de l'auteur derrière le personnage, ainsi que le soulignait déjà John Fiske :

La culture populaire [...] est bien consciente du fait que ses objets sont issus d'une production industrielle et qu'ils n'ont pas, ainsi, le statut d'objet d'art unique. C'est pourquoi ils peuvent être retravaillés, réécrits et complétés d'une manière productive, et qu'ils se prêtent à une participation extérieure comme ne le fait aucun objet d'art achevé. (Fiske $1992: 47$, trad. Saint-Gelais $2011: 376$, note 1)

L'« école italienne », qui réalise une grande partie de la production de bandes dessinées Disney, est tout à fait représentative de ce phénomène : des équipes de scénaristes et de dessinateurs travaillent simultanément sur un même personnage sans que leur nom soit mis en avant. Par exemple, les aventures de Fantomiald (identité secrète de Donald qui parodie les super-héros), qui sont parues en France dans Mickey Parade, ont été rassemblées dans plusieurs volumes chez Glénat (2014-2016), mais le nom des auteurs n'apparaît pas sur la couverture. Les personnages Disney circulent aisément d'un auteur de $\mathrm{BD}$ à l'autre : Carl Barks hérite du personnage de Donald, à qui il donne une famille (Picsou, Riri, Fifi, Loulou), qui est ensuite récupérée par des dizaines de continuateurs, dont Don Rosa, dans une logique d'expansion indéfinie de l'univers des Canards Disney. Mais ce système de production industrielle n'est pas incompatible avec l'émergence d'œuvres et d'auteurs "cultes", à l'instar de La Jeunesse de Picsou de Don Rosa, initialement publiée en douze livraisons chez l'éditeur danois Egmont entre 1992 et 1994, et dont la reconnaissance institutionnelle est marquée en France par la parution d'une 
édition luxueuse chez Glénat. L'étude de ses diverses versions permettra de faire apparaitre les variations induites par le glissement du feuilleton au chapitre en bande dessinée. Au niveau socio-culturel, le chapitrage s'inscrit dans une totalité achevée et définitive, qui rompt avec le caractère éphémère de la publication en magazine. Le dispositif chapitral de La Jeunesse de Picsou s'accompagne, dans les œuvres complètes de Don Rosa parues chez Glénat, d'un appareil paratextuel qui contextualise le travail de l'auteur ou apporte des informations supplémentaires sur le monde fictionnel: chaque chapitre est suivi par une section intitulée «Vues d'auteur » écrite par Don Rosa, qui livre toutes les informations sur la réalisation des planches, les références à Barks ou les sources historiques. Elle satisfait ainsi le désir encyclopédique des fans d'accumuler des connaissances intradiégétiques aussi bien qu'extradiégétiques sur l'univers de Picsou. On observe dès lors un renversement de l'« émancipation transfictionnelle du personnage " (Saint-Gelais 2011: 337) : les fans ne recherchent pas tant de nouvelles aventures de Picsou que l'œuvre d'un auteur identifié, qui devient ainsi un objet de collection. J'étudierai le chapitrage des aventures de Picsou à la lumière de la double logique qui le détermine : reconnaissance symbolique d'une œuvre spécifique d'une part, instauration d'une continuité narrative dans l'univers des canards Disney d'autre part. Il s'agira également de ne pas perdre de vue les conséquences sur la lecture des différents choix éditoriaux au cours de l'histoire complexe de la publication de La Jeunesse de Picsou. En effet, l'expérience du récit se trouve sensiblement modifiée par la disparition de la rupture temporelle que suppose la publication feuilletonesque au profit de la seule rupture spatiale du chapitre.

\section{Une série « fanfictionnelle » qui s'inscrit dans un univers qui la déborde}

\section{Une histoire éditoriale foisonnante}

3 Dans une perspective de multiplication des profits, les livraisons de La Jeunesse de Picsou ont été publiées à de multiples reprises dans le monde entier ${ }^{1}$. Pour avoir une idée de l'ampleur du phénomène, on peut se référer à la liste des publications de l'épisode 1 , telle qu'elle est recensée sur la base de données I.N.D.U.C.K.S. (Archives mondiales des publications Disney), qui appelle deux remarques ${ }^{2}$.

D'abord, la publication initiale a lieu au Danemark. Don Rosa rencontre en effet des problèmes représentatifs des auteurs Disney, qui travaillent souvent en free-lance et naviguent entre les maisons d'éditions qui possèdent la licence Disney. En 1986, il est embauché par Gladstone, qu'il quitte en 1989 du fait des conditions de travail peu favorables aux auteurs. Disney refuse en effet de leur accorder la propriété des planches originales, ce qui les prive d'un complément de revenus indispensable. Don Rosa se retrouve alors dans une condition financière précaire, mais fort de l'immense succès qu'ont rencontré ses premiers récits en Europe, et il est embauché en 1990 par le plus gros éditeur européen de bandes dessinées sous licence Disney, Egmont, qui est basé au Danemark. C'est pour Egmont qu'il réalise à partir de 1991 son œuvre maitresse, La Jeunesse de Picsou. La publication initiale a donc lieu dans une langue que l'auteur ne parle pas, mais les planches des douze livraisons sont diffusées peu de temps après dans les magazines américains et européens. La série connaît un succès immédiat et accède à une reconnaissance symbolique majeure en 1995, avant même la fin de sa diffusion aux États- 
Unis, lorsqu'elle se voit attribuer le prix Eisner de la meilleure histoire à suivre. À l'instar de Carl Barks avant lui, le nom de Don Rosa est très rapidement identifié par ses jeunes lecteurs et devient un argument de vente pour les éditeurs. Son style est en effet immédiatement reconnaissable et se caractérise notamment par un goût maniaque pour les détails, une précision historique quasiment maladive, des intrigues échevelées, et un dessin qui doit beaucoup aux fanzines underground des années $1970^{3}$.

On notera que la France est le pays où La Jeunesse de Picsou a été le plus souvent publiée devant les États-Unis. Je m'appuierai essentiellement sur les publications françaises, tout en mobilisant ponctuellement les éditions scandinaves ou américaines à titre de comparaison. On recense plusieurs types de publications :

6 - Le magazine mensuel Picsou Magazine, où les douze livraisons sont publiées une première fois dès qu'elles sont traduites, puis à de multiples reprises les années suivantes.

- Les hors-série de Picsou Magazine, Les Trésors de Picsou, qui compilent les livraisons à plusieurs reprises (en 1998, en 2004 puis en plusieurs volumes dans une publication en cours depuis 2016), pour un budget accessible (29 Francs en 1998, 4,90 Euros en 2004). Le hors-série de 2004 reprend une couverture quasiment identique à celle de 1998, à quelques exceptions notables : l'œuvre est identifiée comme une « série culte » et le nom de l'auteur apparait en gros caractères.

- L'intégrale Don Rosa en sept volumes parue chez Glénat entre 2012 et 2016. La Jeunesse de Picsou correspond aux deux premiers volumes sur les sept qui composent la collection: c'est dans cette édition luxueuse qu'apparaît le chapitrage pour la première fois en France. Elle fait suite aux intégrales également chapitrées parues aux États-Unis.

\section{Instauration d'une continuité dans l'univers des Canards}

$7 \quad$ La Jeunesse de Picsou se présente comme la reconstitution de la biographie du canard le plus riche du monde à partir des allusions trouvées dans les récits de Carl Barks, qui a créé le personnage en 1947. Don Rosa en fait un héros épique moderne et un archétype du "self made man », qui part d'Écosse pour chercher fortune aux États-Unis. Il s'agit donc de ce qu'on appelle, dans le vocabulaire de la culture médiatique, un prequel, qui narre des événements antérieurs aux récits originels ${ }^{4}$. On peut distinguer trois étapes dans la création de La Jeunesse de Picsou :

8 1. Tout d'abord, Don Rosa fait le relevé exhaustif de toutes les allusions à la jeunesse de Picsou dans les bandes dessinées que Barks a consacrées au personnage, ce qui lui permet d'établir une timeline de la vie de Picsou :

Quel défi, pour un fan inconditionnel, de prendre en compte tous les «détails» concernant les débuts de la vie de Picsou qui jalonnaient les histoires classiques de Carl Barks, quel que soit la longueur de ces fragments d'histoire ou l'obscur obscurcissement qu'ils avaient pu subir! Si Picsou a fait un commentaire à propos de sa jeunesse dans la troisième bulle de la cinquième case de la septième page de la deuxième histoire d'un certain comic book de 1957 - pourvu que ce soit une histoire écrite par Carl Barks -, alors ce fait est mentionné quelque part dans la série. (Don Rosa 2012:6)

9 Par exemple, l'acquisition des lorgnons en 1895, mentionnée par Picsou dans un récit de Barks paru dans Uncle $\$$ crooge en $1958^{5}$, apparaît dans le chapitre $5^{6}$. De même, Don Rosa consacre le chapitre $7^{7}$ à un voyage en Australie signalé par Barks ${ }^{8}$. Don Rosa apporte parfois de légères modifications aux données barksiennes pour des raisons de précision historique : afin de coïncider avec les dates de la ruée vers l'or, la rencontre avec Soapy 
Slick et Goldie a lieu en 1896 dans le chapitre 8, «Le Prospecteur de la vallée de l'Agonie Blanche ${ }^{9}$ ", et non en 1898, comme c'était le cas dans une histoire de Barks, « Au nord du Yukon $^{10} »$. L'hypotexte barksien est revendiquée par Don Rosa à travers la dédicace «D.U.C.K.» («Dedicated to Uncle Carl from Keno»), systématiquement cachée dans les premières cases des épisodes et dans les couvertures des comic books qui sont reprises en en-tête des chapitres.

10 2. À partir de ces éléments de départ, il constitue un plan préparatoire détaillé constitué de douze parties, chacune d'entre elles correspondant à une période de la vie de Picsou, entre 1877 et 1947. Ainsi, le but de la première partie était d'« inclure chaque "info barksienne" où il était question de la jeunesse de Picsou et de ses ancêtres » (Don Rosa $2012: 26)$.

11 3. Enfin, les planches sont réalisées au fur et à mesure, en vue de la publication chez Egmont. Don Rosa consacre également d'autres épisodes à la jeunesse de Picsou, mais il s'agit de projets autonomes, publiés ultérieurement dans différents magazines. Je reviendrai ultérieurement sur la numérotation problématique de ces épisodes et de leur version chapitrale.

Plus qu'un simple hommage à l'auteur fétiche de son enfance, l'œuvre de Don Rosa se caractérise par la volonté, inédite chez Disney, « d'établir une continuité dans l'univers des canards de Disney au sens où l'entendent les comic books» (Chelebourg 2013 : 112). Alors que les récits de Barks étaient pensés sur un modèle sériel (à savoir, des histoires autonomes qui ne sont pas situées temporellement), La Jeunesse de Picsou transforme l'univers des Canards en cycle en instaurant une continuité narrative et chronologique dans les aventures de Picsou ${ }^{11}$. Plus que la logique genettienne du rapport entre un hypotexte et un hypertexte (Genette 1991), c'est la logique expansionniste de la transfictionnalité, telle qu'elle a été théorisée par Saint-Gelais (2011), qui semble pertinente pour aborder La Jeunesse de Picsou, dans la mesure où les rapports entre l'œuvre source et l'œuvre dérivée s'établissent au niveau de l'univers fictionnel :

Il y a transfictionnalité lorsque deux textes ou davantage "partagent» des éléments fictifs (c'est-à-dire, y font conjointement référence), que ces éléments soient des personnages, des (séquences d')événements ou des mondes fictifs; quant aux «textes", il peut s'agir aussi bien de textes au sens strict (romans, nouvelles, mais aussi essais dans certains cas) que de films, bandes dessinées, épisodes télé, etc. (Saint-Gelais 2012 : n. p.)

13 À ce titre, La Jeunesse de Picsou se rapproche à certains égards de la pratique de la fanfiction: on a pu avoir un aperçu du recours systématique au vocabulaire fanique par Don Rosa dans les citations relevées plus haut. Dans cette perspective, l'un des principaux enjeux de La Jeunesse de Picsou est de respecter le plus fidèlement possible les faits fictionnels établis par les récits de Barks : « pas question de modifier l'univers des canards de Barks » (Don Rosa 2012: 7). Il instaure une hiérarchie dans l'univers des canards : l'œuvre de Barks est considérée comme canonique, tandis que Don Rosa se met sur un pied d'égalité avec les autres continuateurs:

Je considère comme apocryphe toute aventure de Picsou n'ayant pas été écrite par Carl Barks sur ce personnage qu'il a créé. (Donc je suppose que les miennes le sont aussi. Pas de traitement de faveur...). Mais à chaque fois qu'un vieux maître de mon enfance avait déjà établi un élément concernant l'enfance de Picsou, j'ai essayé de le laisser me guider. (Don Rosa $2012: 27$ 1/2)

14 Par exemple, les cases du premier chapitre, "Le dernier du clan McPicsou», dans lesquelles le jeune Picsou exerce son premier métier de cireur de chaussures, reprennent 
celles d'un autre auteur Disney, Toby Strobl, dans une histoire intitulée «Le Blues du Businessman $^{12}$ ». À l'évidence, La Jeunesse de Picsou n'est cependant pas une fanfiction à proprement parler. En effet, tandis que les fanfictions sont extérieures aux industries qui régulent la production canonique, la légitimité que procure à Don Rosa la maison d'édition Egmont, qui détient le copyright Disney, institutionnalise d'emblée la canonisation de l'œuvre. Mais son travail personnel n'est pas détaché du reste de la communauté fanique, dont il sollicite ponctuellement la collaboration :

afin de rendre la série la plus «légitime» possible, ma première tâche a été de recenser tous les obscurs détails «barksiens » à propos de Picsou que nous autres, fans de Canards, connaissons depuis si longtemps. Puis j'ai assemblé ces événements dans une continuité, que j’ai fractionnée en douze segments logiques, chacun traitant une période de la vie de Picsou telle que l'a traitée M. Barks. Et puisque - comme je l'ai dit - Picsou n'est pas plus ma propriété que celle de n'importe quel autre passionné de Canards, j'ai envoyé une copie de ce schéma aux fans les plus connus du monde entier, sollicitant leurs commentaires et leur aide. (Don Rosa 2012:8)

On retrouve bien, dans ce commentaire, la dynamique spécifique de la création fanique : Don Rosa s'appuie sur une connaissance encyclopédique de l'œuvre de Barks qui lui permet de constituer un "fanon ", c'est-à-dire un univers parallèle construit à partir des indices des récits d'origine. Comme l'a noté Saint-Gelais, "le fanon, fréquemment, réinjecte en fiction ses résultats » $(2011: 412)$. Les contraintes narratives de La Jeunesse de Picsou sont celles auxquelles sont confrontés les prequels de manière générale, qui, « pour ne pas trahir l'attente des fans, doivent respecter la cohérence de l'œuvre et de l'intrigue originelles tout en proposant une intrigue suffisamment dynamique pour renouveler l'intérêt, avec le handicap conséquent que son issue est connue à l'avance par les lecteurs " (Lemonnier $2003:$ 10, cité par Saint-Gelais 2011: 80). Le récit transfictionnel dérive ainsi de lacunes qui n'étaient pas exploitées par la mise en intrigue des récits source et qui n'étaient pas censées éveiller la curiosité ou le suspense (Saint-Gelais 2011: 77). Mais pour le public le plus investi, ces zones indéterminées du récit sont un prétexte à la spéculation, voire à la construction de nouvelles intrigues. Les prequels reposent sur deux principes parfois contradictoires: une fidélité maximale à l'œuvre d'origine et le maintien d'une cohérence diégétique alors que les récits source ne sont pas toujours cohérents eux-mêmes. Saint-Gelais a ainsi souligné la «pulsion suturante » qui anime le fanon:

face à un espace transfictionnel profus et segmenté en nombreux épisodes [...] la stratégie interprétative dominante consiste à jouer la carte de la continuité, de la cohérence globale de l'ensemble - quitte à prendre en défaut ses parties constituantes lorsque celles-ci s'ajustent imparfaitement. (Saint-Gelais 2011 : 413)

Dans ce sens, la résolution de paradoxes constitue la matière principale de l'intrigue de certains chapitres de La Jeunesse de Picsou, comme le chapitre $5^{13}$ :

Ce chapitre a été en partie conçu pour résoudre l'un des très rares problèmes que me posaient les « infos » sur Picsou de Barks révélées dans ses nombreuses histoires classiques. En la circonstance, comment Picsou pouvait-il être né dans une famille pauvre tout en étant l'héritier d'un magnifique château ancestral dans les Highlands d'Écosse ? J'avais lancé des pistes en vue de la résolution de son paradoxe dans mon premier chapitre, et selon le plan que j'avais patiemment mis au point pour la série, je savais que je règlerais cette question ici, au chapitre 5. (Don Rosa 2012 : 120)

L'instauration d'une continuité narrative qui opère une suture entre des indices contradictoires découverts dans les récits de Barks est révélatrice, dans le sens où elle 
informe la disposition chapitrale de l'œuvre. Les histoires de Barks se déroulent selon une temporalité sérielle immuable, elles ne sont donc pas chapitrées dans les éditions intégrales de Glénat, contrairement à celles de Don Rosa. Pour autant, la logique sérielle a tout de même interféré avec le chapitrage de La Jeunesse de Picsou, qui est l'aboutissement d'une logique éditoriale complexe.

\section{Épisodes, chapitres et continuité narrative}

\section{Les douze livraisons initiales}

La désignation des différentes formes de segmentation du récit en épisodes ou en chapitres apparaît explicitement à plusieurs endroits dans les différentes éditions :

19 - dans le péritexte éditorial (sommaires, pages de présentation), qui ne dépend pas toujours du choix de l'auteur, sauf dans le cas des récentes éditions en volumes, qui ont très largement été dirigées par Don Rosa ;

- dans le sous-titrage de la première case des livraisons, qui fait quant à lui partie intégrante du dessin des planches.

Lorsqu'on compare les différentes publications, l'instabilité terminologique du dispositif apparait nettement. La publication initiale danoise fait apparaître le mot «kapitel» (" chapitre ${ }^{14} »$ ), ce qui s'explique certainement par le fait que la commande à Don Rosa était pour une histoire unifiée en douze livraisons : le modèle littéraire est donc présent dès la première publication en magazine, mais il tend à se dissoudre dans les diffusions suivantes. La nomenclature est plus aléatoire en anglais et en français. Dans les versions en anglais, la case-titre des douze premières livraisons, qui imitent l'esthétique de l'album photo, sont systématiquement sous-titrées " parties » (« parts»). La nomenclature est ensuite rétablie dans la table des matières des versions livresques. Comparons quelques versions du chapitre $8^{15}$ pour voir ce qu'il en est dans les traductions françaises:

- dans la publication française initiale ${ }^{16}$, la case-titre est modifiée afin de faire disparaitre le texte anglais, et c'est le terme " épisode » qui est retenu, sans indication de titre, ni au début de la planche, ni dans le sommaire, contrairement à la version américaine.

- dans le premier hors-série, qui publie l'ensemble des douze livraisons de La Jeunesse de Picsou, Les Trésors de Picsou $\mathrm{n}^{\circ} 1 \mathrm{H}$ (avril 1998), la première planche est identique à celle de Picsou Magazine, mais le titre est indiqué dans la page introductive qui précède l'épisode : "L'empereur du Klondike» (traduction du titre original «King of the Klondike»). Le sommaire n'affiche aucun titre ni aucune désignation, mais une simple numérotation.

- Le choix du terme « chapitre » n'est arrêté que dans l'intégrale de Glénat, qui est celle que l'auteur conçoit comme définitive, en cohérence avec le modèle romanesque dont le format livresque est issu. La case initiale est rétablie dans sa version originale en anglais. Un nouveau titre est arrêté : « Le Prospecteur de la vallée de l'Agonie blanche » et la date à laquelle se déroule l'aventure est signalée (1896-1897), alors qu'Egmont n'avait pas autorisé Don Rosa à dater les aventures de Picsou, certainement pour se conformer à la tradition sérielle de la bande dessinée, dans laquelle les personnages évoluent dans un temps immuable :

mes responsables éditoriaux de chez Egmont ne m'ont pas permis de mentionner des années précises pour mes scénarios. [...] Mais j'ai trouvé moyen de donner des indications précises sur le moment où mon histoire se passait. Quelquefois, je 
plaçais un calendrier dans le décor pour montrer la date, en espérant que les

éditeurs ne le verraient pas. (Don Rosa 2012 : 102)

Il est intéressant de noter que l'ensemble des publications françaises accentue l'autonomie des épisodes plutôt que la continuité narrative qui régit l'ensemble: ainsi, tandis que les versions américaines s'achèvent sur une clausule " ouvrante » (Hamon 1975) héritée de la tradition feuilletonesque, "To be continued ", on trouve en France une clausule «fermante »: «Fin ». Ces variations s'expliquent en partie par l'adaptabilité du format: pour emprunter au vocabulaire télévisuel, La Jeunesse de Picsou est une "série feuilletonnante » avec une histoire continue, mais qui préserve une certaine autonomie des épisodes, caractérisés par leur unité géographique (Écosse, Mississipi, Australie...) et un marquage temporel précis (1877, etc.). Le conflit entre les péritextes auctorial et éditorial s'explique peut-être également par la différence de contexte entre le comic book américain et le magazine français. Les lecteurs de Picsou Magazine ne sont pas tous abonnés et n'accèdent pas systématiquement à tous les numéros, qu'ils se procurent irrégulièrement en fonction du bon vouloir de leurs parents ou des sommes d'argent de poche dont ils disposent. Cependant, l'édition Glénat corrige parfois ce type de décisions éditoriales pour proposer une version plus fidèle à celle de l'auteur. Ainsi, le dénouement du chapitre 8 relate un événement fondamental: Picsou découvre la pépite d'or qui le rend enfin riche, après bien des tentatives qui se sont soldées par des échecs. Don Rosa fait ainsi indirectement référence au triangle de Freytag ${ }^{17}$ dans l'analyse qu'il en propose :

C'est le «point culminant » de la série en termes shakespeariens (si je me souviens correctement de mon cours de littérature anglaise à l'université). En tant que tel, ce n'est pas la fin, mais le moment décisif de la vie de Picsou. Dans cette nomenclature littéraire, les chapitres 1 à 7 correspondaient à « la montée de l'action » et les 9 à 12 représentent "l'action retombante", quand Picsou commence à se rendre compte que posséder une fortune n'est pas aussi enthousiasmant que l'acquérir. (Don Rosa $2012: 186)$

Suivant cette construction narrative, le mot «Fin» qui clôturait l'épisode dans Picsou Magazine est remplacé dans le chapitre du livre par «Le commencement.... » (Don Rosa 2012 : 184) qui traduit la clausule de la version américaine, "The beginning... », et qui permet de resituer le chapitre dans la geste épique de Picsou ${ }^{18}$.

Hormis ces quelques modifications, le transfert de l'épisode au chapitre se fait de manière fluide en ce qui concerne les douze livraisons initiales. Entre les diverses publications, les planches elles-mêmes connaissent peu de changements à quelques exceptions près : la colorisation et la traduction sont largement remaniées, et Don Rosa, affranchi de la contrainte de la pagination du comic book, ajoute parfois des planches à certains chapitres, comme le chapitre 12, qu'il rallonge de trois pages et dans lequel il s'amuse à ajouter la mention de son prix Eisner ${ }^{19}$. Mais dans l'ensemble, on peut parler, à la suite de Dionne (2008), de coïncidence du paradispositif épisodique et du dispositif chapitral dans la mesure où les divisions sont identiques ${ }^{20}$. Les choses se compliquent lorsqu'il s'agit des chapitres adventices qui les complètent.

\section{Chapitres adventices : une intégration problématique}

Le projet de Don Rosa visait à « offrir au passé de Picsou un cadre qui pourrait être étoffé par des épisodes flashbacks supplémentaires, par [lui]-même ou par tout autre scénariste ou dessinateur » (2012: 7). Il propose ainsi ponctuellement des épisodes autonomes, réalisés entre 1991 et 2006, qui se situent à divers points de la vie de Picsou, ce qui pose 
des problèmes pour le choix de l'ordre de publication dans les éditions intégrales. Dès la publication en magazine, Don Rosa opte pour un système de balisage qui apparait dans la plupart des cases initiales : l'épisode sous-titré dans Uncle $\$$ crooge " part three-and- $a$-half $~^{21}$ , par exemple, publié en magazine en 1998, se déroule entre les épisodes $3^{22}$ et $4^{23}$. Le premier hors-série français ${ }^{24}$ qui compile La Jeunesse de Picsou publie les différents épisodes dans l'ordre de la chronologie diégétique, au détriment des articulations narratives mises en place dans le récit unifié que constituaient les douze premières livraisons. L'épisode 3Bis n'apparaît pas dans cette version : en 1998, il est en effet encore inédit en France, il ne sera publié qu'en 1999. L'édition Glénat opte quant à elle pour une disposition qui préserve l'unité des douze livraisons dans un premier tome, et publie indépendamment dans un deuxième tome les "expansions parallèles" (Saint-Gelais 2011 : 93), en suivant un système de numérotation du type 1B, 2B, etc. qui permet aux lecteurs de situer les chapitres dans la chronologie interne à l'histoire. Les lecteurs peuvent ainsi décider de suivre un parcours de lecture qui respecte strictement la chronologie diégétique en naviguant entre les deux volumes ${ }^{25}$. En outre, si la numérotation des douze premières livraisons demeure fixe, celle des épisodes adventices évolue lorsque de nouveaux segments viennent s'ajouter. Par exemple, l'épisode numéroté 8 Bis dans Les Trésors de Picsou ${ }^{26}$, «Les Deux cœurs du Yukon", devient le chapitre $8 C$ chez Glénat ${ }^{27}$, Don Rosa ayant réalisé en 2006 une histoire qui se déroule antérieurement, intitulée " La Prisonnière de la vallée de l'Agonie blanche ", à laquelle est finalement attribuée la numérotation $8 \mathrm{~B}^{28}$. L'ordre de publication privilégié par Les Trésors de Picsou est d'autant plus difficile à tenir que toutes les histoires adventices ne sont pas des interquels, qui se déroulent entre deux épisodes publiés précédemment : certaines sont des midquels, dont l'action se situe entre le début et la fin d'un épisode. C'est le cas des chapitres $8 \mathrm{~B}$ et $8 \mathrm{C}$, qui approfondissent l'histoire d'amour entre Picsou et Goldie, qui restait implicite dans le chapitre 8 , mais c'est également le cas du chapitre 0 , intitulé «Canards, cents et destinées! " ${ }^{29}$. L'essentiel de l'action se déroule pendant une scène du chapitre $1^{30}$, dans lequel le jeune Picsou gagne son premier sou en cirant des chaussures. Il s'agit d'un exemple de restauration d'une continuité "défaillante» chez Barks: le chapitre tente d'expliquer pourquoi le «sou fétiche» (la première pièce gagnée par Picsou lorsqu'il était enfant) est une pièce américaine alors qu'il l'a obtenue en Écosse, suivant le paradoxe formulé par les petits-neveux de Picsou :

Riri : - Mais... C'est une pièce américaine!

Fifi : - Et toi tu vivais en Écosse !

Picsou : - Oui. Je n'ai jamais compris comment elle est arrivée là.

(Don Rosa 2013 [1996] : 10)

26 L'intrigue est recentrée sur un personnage absent du premier épisode (on peut parler de «transfocalisation» dans la terminologie genettienne ${ }^{31}$ ) et offre une variation sur le motif du paradoxe temporel : la sorcière Miss Tick remonte dans le temps pour essayer de subtiliser le sou fétiche, mais c'est finalement elle qui est contrainte de le donner au jeune Picsou. De nombreuses cases représentent des passages identiques, mais en modifiant l'ocularisation, pour reprendre la typologie de François Jost (1987). Du point de vue de la chronologie de la production, l'étiquette de midquel est un peu impropre car cette histoire a été écrite avant le chapitre 1, même si elle n'a été publiée que plusieurs années après, comme le rappelle le texte de présentation qui accompagne l'épisode dans la dernière édition de La Jeunesse de Picsou dans les Trésors de Picsou :

$\mathrm{Ne}$ nous trompons pas: bien que qualifiée d'épisode 0 , cette histoire n'a été dessinée que trois ans après le premier épisode, bien que Don Rosa ait commencé à 
travailler dessus avant même de débuter La jeunesse de Picsou! Par ailleurs, les évènements qu'elle décrit ne précèdent ni ne suivent ceux de l'épisode un, mais se déroulent pendant une scène de celui-ci! Tu suis? Bien... (Les Trésors de Picsou. La Jeunesse de Picsou, vol.1, 2016 : 154)

Il s'agit donc d'un midquel si on se place du côté du lecteur, qui a eu accès à l'histoire en magazine plusieurs années après l'épisode 1 , ou qui lit les chapitres dans l'ordre de publication proposé par l'édition Glénat. En ce qui concerne la logique narrative, il est plus pertinent de lire le chapitre 0 après l'épisode 1 , qu'il étoffe en adoptant le point de vue d'un personnage absent. La première édition des Trésors de Picsou ${ }^{32}$ le place donc à la fin du volume, comme un épisode bonus décorrélé de l'intrigue principale. L'édition la plus récente a publié dans son premier volume, sorti en $2016^{33}$, trois épisodes de $L a$ Jeunesse de Picsou accompagnés des histoires de Barks qui les ont inspirées. Dans cette version, l'épisode 0 est proposé après l'épisode 1 , mais avant l'épisode 2 . Dans les éditions américaines, les premières cases des histoires adventices publiées ultérieurement ne sont initialement pas numérotées, mais le chapitrage apparait en couverture du comic book ${ }^{34}$.

Un autre cas intéressant est celui de l'histoire bonus intitulée «Le Rêve de toute une vie ! $»^{35}$. Grâce à une machine qui permet aux psychiatres d'observer les rêves de leurs patients, les Rapetou pénètrent dans un rêve de Picsou afin d'essayer de lui soutirer la combinaison de la porte de son coffre-fort. Donald est à son tour envoyé dans le cerveau de Picsou afin de contrecarrer les plans des Rapetou. Il s'agit de ce que Saint-Gelais nomme un «contrefictionnel » (2011: 166), c'est-à-dire une réécriture qui modifie le cours de la fiction en en proposant une version contradictoire. Il permet de faire vivre à Picsou le happy ending qu'il n'a jamais connu avec Goldie, sans compromettre le canon fictionnel établi précédemment, dans lequel il se sépare de celle-ci pour continuer de courir le monde. Ce bonus n'est donc pas un contrefictionnel radical, puisqu'il se déroule dans un rêve. Il se rapproche de la notion de « disnarré » chez Gerald Prince (1988), qui désigne les événements qui n'ont pas eu lieu, mais qui auraient pu avoir lieu, et auquel le récit fait référence de manière négative ou hypothétique. Mais son statut particulier l'empêche d'être inclus dans une numérotation suivie.

29 Le chapitrage final correspond donc mieux à l'intention auctoriale que le découpage épisodique, puisqu'il permet de restituer la continuité narrative des douze livraisons de La Jeunesse de Picsou, dont la rédaction a été minutieusement planifiée :

Il s'agissait d'«une» histoire continue racontée en douze chapitres. Une fois rassemblée en une seule belle édition, cette collection d'histoires a donné un magnifique tome dont je suis très fier. (Don Rosa, $2013: 4$ )

30 Selon l'auteur, La Jeunesse de Picsou trouve donc son véritable aboutissement dans la version livresque qui permet de mettre en avant la continuité narrative, notamment par le biais du découpage chapitral et qui permet également de réintégrer les épisodes adventices dans une numérotation homogène. Le chapitrage est bien ici le "dispositif " (Dionne 2008) qui marque la fixation de l'œuvre après l'étape transitoire de la publication en livraisons (" paradispositif»). Mais il instaure aussi une forme de discontinuité dans le flux narratif de la bande dessinée. En l'occurrence, cette discontinuité est redoublée par le retour réflexif qui succède aux chapitres et qui contribue à enrichir l'univers fictionnel. En effet, la segmentation est investie par la fonction documentaire de ces «Vues d'auteurs » qui rompent l'immersion dans le récit pour enrichir l'univers fictionnel.

31 Le chapitrage s'inscrit donc dans une perspective qui revalorise la figure de l'auteur et institutionnalise la dimension «culte» de La Jeunesse de Picsou. Cette notion, issue des 
catégories religieuses, est cependant à mobiliser avec prudence, comme l'ont rappelé Philippe Le Guern (2002: 18) ou Éric Maigret (2002: 97-110). Le culte médiatique résulte d'une « organisation de la rareté artistique » (Le Guern 2002:17) qui, dans une certaine mesure, n'est pas si éloignée de l'esthétique romantique, mais qui s'en distingue par le fait que les œuvres qu'elle isole sont issues d'un système de production industrielle. Pour Le Guern, il ne s'agit pas, pour les publics, d'assimiler à la grande culture les œuvres identifiées comme "cultes", mais plutôt d'ériger leurs défauts en qualités (notion de « kitsch », séries B, etc.). Toutefois, dans le cas qui m'occupe, ce n'est pas cette logique qui est à l'œuvre. Les critères évaluatifs appliqués sont proches de la hiérarchie de valeurs inhérente à la culture légitime, notamment à travers l'isolement de traits stylistiques distinctifs qui singularisent l'auteur.

Ce qui est très frappant, c'est que ce processus est entamé dès les premières publications de Don Rosa, et qu'il est dû à la fois aux éditeurs de magazines, mais aussi au public enfantin qui distingue immédiatement les bandes dessinées de Don Rosa parmi la production pléthorique de Disney. Comme je l'ai signalé plus tôt, le terme "culte » apparait sur la couverture du hors-série datant de 2004, les éditeurs prenant ainsi acte du succès de l'œuvre. D'une certaine manière, on peut dire que Picsou Magazine est d'emblée une publication distinctive au sens bourdieusien du terme (Bourdieu 1979), puisqu'elle propose dans ses pages une documentation contextuelle, là où d'autres magazines Disney, comme Super Picsou Géant ou Mickey Parade, diffusent uniquement les bandes dessinées. Le nom de l'auteur est mis en avant dès les publications en magazine, et ce processus aboutit à la possibilité pour Don Rosa de contrôler les éditions intégrales de son œuvre et de participer à sa propre patrimonialisation tout en reconstituant la continuité narrative du récit par le biais du chapitrage. Le calendrier de la publication des volumes correspond à une date où Don Rosa a atteint un certain âge et l'impression d'une forme d'achèvement de sa carrière qui lui permet de publier ses œuvres intégrales, mais aussi où les premiers lecteurs de ses récits ont atteint l'âge adulte (et avec un peu de chance, ont acquis le pouvoir d'achat qui va avec) : restés attachés à un auteur qui a marqué leur enfance, ils sont prêts à investir dans une édition de luxe et à assumer la différence de prix substantielle par rapport aux Trésors de Picsou (30 euros le volume, alors que le premier hors-série coûtait 30 Francs). En effet, tandis que Picsou Magazine est avant tout destiné à des enfants, l'édition Glénat s'adresse - comme cela apparait nettement dans les propos de l'auteur - à des fans adultes qui ont déjà lu ces histoires quand ils étaient jeunes. Le format livresque et le dispositif chapitral qu'il induit donne à La Jeunesse de Picsou la légitimité symbolique du graphic novel, et autorise une lecture complète et répétée ouvrant la voie à une activité interprétative plus dense que la consultation des épisodes dans des magazines épars. Le prix coûteux de cette édition est justifié par sa valeur ajoutée : qualité de la couverture, du papier, de la colorisation et de la traduction, et travail documentaire fourni. On peut noter cependant une forme de "conservatisme" (sans connotation morale) des fans qui se plaignent souvent auprès de l'auteur des modifications des planches :

je me donne un mal de chien pour m'assurer que toutes ces planches soient recolorisées de façon réaliste, dramatique, ou complexe comme je l'avais toujours voulu, et le résultat me ravit. Mais voilà ces lecteurs qui me disent préférer les anciennes versions ! Ce à quoi je réponds : « Mais... mais ces nouvelles colorisations sont tellement meilleures! [...]» Ce à quoi ils rétorquent: «Mais elles étaient comme ça quand j'étais petit, c'est comme ça que je les aimais. »

Eh bien, comme je crois véritablement en la beauté de la nostalgie, je n'ai pas de réponse face à un tel raisonnement. Revivre son enfance est une chose magnifique. 
Mais c'est aussi une chose magnifique pour un auteur ou un artiste de voir son

travail présenté à son public tel qu'il l'avait voulu. (Don Rosa $2013: 6$ ) une œuvre «culte", mais également le lien affectif qui unit le public au récit. Ce goût pour les éditions d'origine, qui se situent au plus près les émotions ressenties pendant l'enfance, reflète la morale quasiment proustienne de La Jeunesse de Picsou : les trésors les plus précieux de Picsou ne sont pas ses richesses, mais ses souvenirs. Ainsi, les versions imparfaites, inachevées, lues dans un ordre aléatoire, remportent-elles parfois davantage l'adhésion des lecteurs que la version refondue sur le modèle littéraire.

\section{BIBLIOGRAPHY}

\section{Ouvrages critiques}

Besson, Anne (2004), D’Asimov à Tolkien: cycles et séries dans la littérature de genre, Paris, CNRS Éditions, coll. «CNRS littérature ».

Bourdieu, Pierre (1979), La distinction. Critique sociale du jugement, Paris, Éditions de Minuit, coll. «Le sens commun ».

Chelebourg, Christian (2013), Les Fictions de jeunesse, Paris, Presses Universitaires de France, coll. «Les Littéraires».

Dionne, Ugo (2008), La voie aux chapitres : poétique de la disposition romanesque, Paris, Seuil, coll. « Poétique ».

Fiske, John (1992), «The Cultural Economy of Fandom », in The Adoring Audience : Fan Culture and Popular Media, L. A. Lewis (dir.), Londres, Routledge, p. 30-49.

von Freytag, Gustav, Gesmmelte Werke, Bd. 14, Die Technik des Dramas, Leipzig, Hirzel.

Genette, Gérard (1972), Figures III, Paris, Seuil, coll. « Poétique ».

Genette, Gérard (1982), Palimpsestes : la littérature au second degré, Paris, Seuil, coll. « Poétique ».

Genette, Gérard (1991), Fiction et diction, Paris, Seuil, coll. « Poétique ».

Jost, François (1987), L'œil-caméra : entre film et roman, Lyon, Presses Universitaires de Lyon, coll. " Linguistique et sémiologie».

Hamon, Philippe (1975), « Clausules », Poétique, n²4, p. 495-526.

Le Guern, Philippe (dir.) (2002), Les Cultes médiatiques. Culture fan et œuvres cultes, Rennes, Presses Universitaires de Rennes, coll. « Le sens social ».

Lemonnier, Grégory (2003), Les encyclopédies imaginaires dans les cycles de science-fiction, mémoire de maîtrise, Université de Rennes 2.

Maigret, Éric (2002), « Du mythe au culte... ou de Charybde en Scylla ? Le problème de l'importation des concepts religieux dans l'étude des publics des médias », in Les cultes médiatiques. Culture fan et œuvre culte, P. Le Guern (dir.),Rennes, Presses Universitaires de Rennes, p. 97-110.

Cahiers de Narratologie, 34 | 2018 
Prince, Gerald (1988), « The Disnarrated », Style, n²2(1), p. 1-8.

Saint-Gelais, Richard (2011), Fictions transfuges : la transfictionnalité et ses enjeux, Paris, Seuil, coll. « Poétique ».

Saint-Gelais, Richard \& Frank Wagner (2012), « Fictions transfuges : la transfictionnalité et ses enjeux. Entretien avec Richard Saint-Gelais », Vox-Poetica, en ligne, consulté le 27 septembre 2018, URL : http://www.vox-poetica.org/entretiens/intStGelais.html

\section{Bandes dessinées}

Barks, Carl (1967 [1964]), « La Folle ruée vers l'or lunaire » [« The Loony Lunar Gold Rush »], Le Journal de Mickey, $\mathrm{n}^{\circ} 809$ [Uncle \$crooge $\mathrm{n}^{\circ} 49$ ].

-(1978 [1958]), « Un puits de dollars » [« The Money Well »], Le Journal de Mickey, ${ }^{\circ} 1362$ [Uncle \$crooge $\left.\mathrm{n}^{\circ} 21\right]$.

-(1968 [1960]), « Au nord du Yukon » [« North of The Yukon »], Le Journal de Mickey, n826 [Uncle \$crooge $\left.n^{\circ} 59\right]$.

-(2012-2017), La Dynastie Donald Duck. Intégrale Carl Barks, 24 tomes, Paris, Glénat.

Collectif Disney (2014-2016), Fantomiald [Paperinik], d'après le personnage créé par Guido Martina \& Giovan Battista Carpi, Paris, Glénat, 3 tomes.

Don Rosa, Keno Don (1992-2006), La Jeunesse de Picsou [The Life and Times of Scrooge McDuck], Picsou Magazine, $\mathrm{n}^{\circ} 266-417$, Paris, Disney Hachette Presse.

-(1994-2006 [1992-2006]), La Jeunesse de Picsou [The Life and Times of Scrooge McDuck], Picsou Magazine, $n^{\circ} 266-417$, Paris, Disney Hachette Presse.

-(1992), Her er dit liv, Joakim, hors-série, Danemark, Egmont.

-(1992-1995), Anders And Co., Danemark, Egmont.

-(1994-2004), Uncle \$crooge, n²85-332, États-Unis, Gladstone Publishing, Gemstone Publishing.

-(1995), Walt Disney Giant, ${ }^{\circ} 1$, États-Unis, Gladstone Publishing.

-(1995-1998), Kalle Anka \& C:o, Suède, Egmont.

-(1998), Les Trésors de Picsou. La Jeunesse de Picsou, hors-série n¹H, Paris, Disney Hachette Presse.

-(2006), Aku Ankka, Finlande, Sanoma.

-(2006), The Life and Time of Scrooge McDuck Companion, États-Unis, Gemstone Publishing.

-(2007), The Life and Time of Scrooge McDuck, États-Unis, Gemstone Publishing.

-(2010), The Life and Times of Scrooge McDuck, 2 tomes, États-Unis, Boom !

-(2012-2016), La Grande épopée de Picsou. Intégrale Don Rosa, 7 tomes, Paris, Glénat.

-(2016), Les Trésors de Picsou, La Jeunesse de Picsou, vol. 1, Paris, Disney Hachette Presse.

Strobl, Tony (2002 [1964]), « Le Blues du Businessman » [« Chairman of The Bored »], Picsou Magazine $n^{\circ} 36,2002$ [Uncle \$crooge $n^{\circ} 50,1964$ ].

\section{APPENDIXES}

\section{Annexe : Premières publications de La Jeunesse de Picsou}




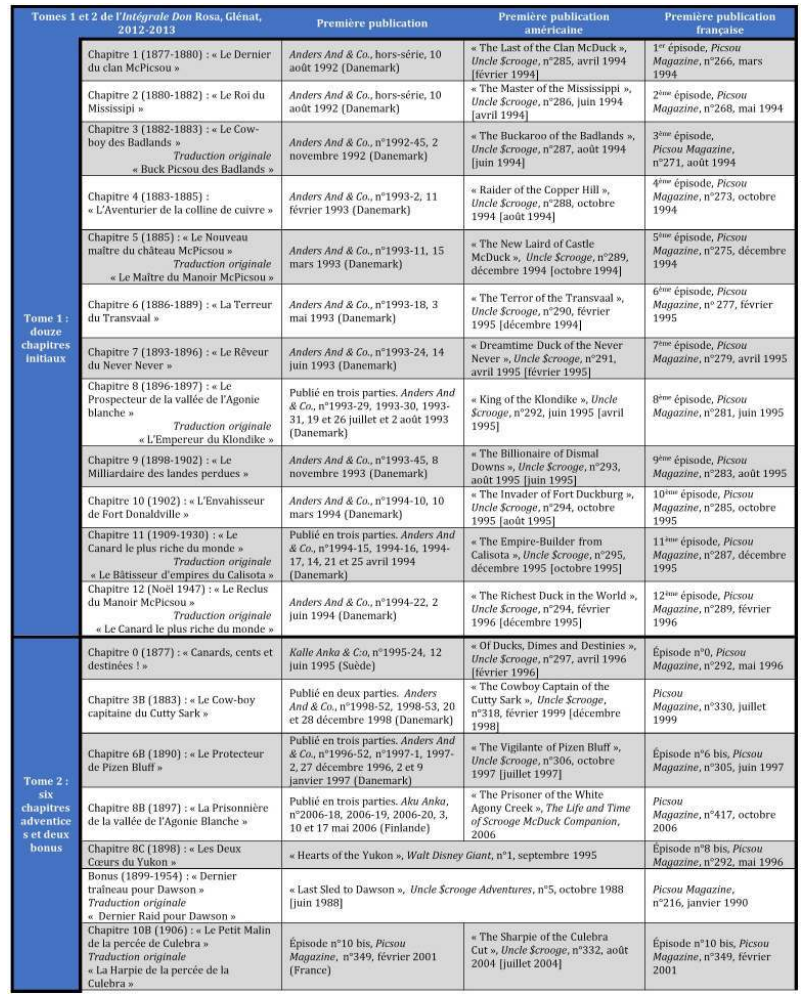

\section{NOTES}

1. Voir le tableau en annexe récapitulant la première publication mondiale, la première publication américaine et la première publication française des différents épisodes.

2. URL : https://inducks.org/story.php?c=D+91308

3. Don Rosa cite souvent Robert Crumb comme source d'inspiration ; il s'agit d'une influence peu commune pour un auteur de bandes dessinées Disney destinées aux enfants.

4. Les prequels ont souvent pour objectif d'étoffer les personnages et leurs relations, auxquels ils s'emploient à donner une «épaisseur » accrue. Ils se rapprochent du flashback ou de l'analepse, que Genette définit comme « une évocation après coup d'un événement antérieur au point de l'histoire où l'on se trouve» (1972: 82). Ainsi, ils sont parfois désignés par l'expression «continuation analeptique».

5. Barks, « Un puits de dollars », Le Journal de Mickey, $n^{\circ}$ 1362, août 1978 [Uncle \$crooge n²1, mars 1958]

6. Don Rosa, chapitre 5, «Le Nouveau Maître du château McPicsou », Intégrale Don Rosa, t. 1, Glénat, 2012 [1993].

7. Don Rosa, chapitre 7, « Le Rêveur du Never Never », Intégrale Don Rosa, t. 1, Glénat, 2012 [1993].

8. Barks, «La Folle ruée vers l'or lunaire », Le Journal de Mickey, n 809, novembre 1967 [Uncle $\$ c r o o g e n^{\circ} 49$, mai 1964].

9. Don Rosa, chapitre 8, «Le Prospecteur de la vallée de l'Agonie Blanche », Intégrale Don Rosa, t. 1, Glénat, 2012 [1993].

10. Barks, " Au nord du Yukon », Le Journal de Mickey, n 826, mars 1968 [Uncle \$crooge $n^{\circ} 59$, septembre 1960].

11. Suivant la distinction entre cycle et série proposée par Anne Besson (2004).

12. Strobl, «Le Blues du Businessman », Le Journal de Mickey, ${ }^{\circ} 699$, octobre 1965 [Uncle \$crooge $n^{\circ}$ 50, juillet 1964]. 
13. Don Rosa, chapitre 5, "Le Nouveau Maître du château McPicsou », Intégrale Don Rosa, t. 1, Glénat, 2012 [1993].

14. Par exemple: Kapitel 4, "Kobberkongen fra Montana ", Anders And \& Co. n¹993-02, février 1993.

15. Voir l'ensemble des différentes planches titres sur le site I.N.D.U.C.K.S. : https://inducks.org/ story.php?c=D+92514

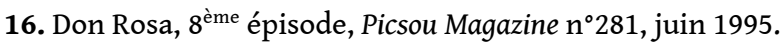

17. Gustav Freytag (1887) schématise la structure idéale de la tragédie sous une forme pyramidale dans laquelle se succède l'introduction, la montée, le climax, la descente et la catastrophe.

18. Don Rosa, part 8, « King of the Klondike », Uncle \$crooge n²92, avril 1995.

19. Don Rosa, chapitre 12, "Le Reclus du manoir McPicsou », Intégrale Don Rosa, t. 1, Glénat, 2012 [1994] : 164-167.

20. Selon Dionne (2008), le dispositif correspond au découpage du roman unifié, tandis que le découpage périodique (livraison, feuilleton...) constitue le paradispositif précédant le rassemblement en volume: "Pour la plupart des romans périodiques et des romans feuilletons (en tout cas, pour ceux que nous lisons encore aujourd'hui), la segmentation paradispositive n'est jamais qu'un état transitoire, postérieur à la genèse proprement dite [...], mais néanmoins toujours vivant, et susceptible de mutations diverses. Ce travail d'ajustement ne s'interrompt vraiment que lors de la cristallisation de l'opus en une forme autorisée, généralement celle du volume : c'est la refonte livresque, la (re)publication post-périodique, qui procède à la fixation romanesque. La segmentation paradispositive n'est donc le plus souvent qu'une étape dans la "carrière" d'un roman (à moins, bien sûr qu'il ne meure au feuilleton...). Les livraisons seront éventuellement, parfois simultanément, voire préalablement refondues, remodelées, de façon à composer un texte "suivi", susceptible d'une lecture continue » (2008:89-90).

21. Don Rosa, "The Cowboy Captain of the Cutty Sark», Uncle \$crooge, n³18, février 1999 [décembre 1998]. En France : Picsou Magazine, n³30, juillet 1999.

22. Don Rosa, «The Buckaroo of the Badlands", Uncle \$crooge, n²87, août 1994 [juin 1994. En

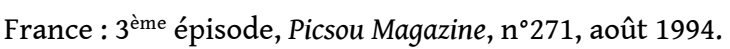

23. Don Rosa, «Raider of the Copper Hill», Uncle \$crooge, n²88, octobre 1994 [août 1994]. En France : $4^{\mathrm{ème}}$ épisode, Picsou Magazine, n²73, octobre 1994.

24. Les Trésors de Picsou. La Jeunesse de Picsou, hors-série n¹H, 1998.

25. C'est également cette disposition qui a été retenue dans l'édition américaine chez Boom! (2010).

26. Les Trésors de Picsou. La Jeunesse de Picsou, hors-série $n^{\circ} 1 \mathrm{H}, 1998$.

27. Don Rosa, chapitre 8C, «Les Deux cœurs du Yukon », Intégrale Don Rosa, t. 2, Glénat, 2013 [1995].

28. Don Rosa, chapitre 8B, « La Prisonnière de la vallée de l'Agonie blanche », ", Intégrale Don Rosa, t. 2, Glénat, 2013 [2006].

29. Don Rosa, chapitre 0, « Canards, cents et destinées ! ", 2013 [1996].

30. Don Rosa, chapitre 1, « Le Dernier du clan McPicsou », 2012 [1992].

31. Genette (1982: 285).

32. Les Trésors de Picsou. La Jeunesse de Picsou, hors-série n¹H, 1998.

33. Les Trésors de Picsou, La Jeunesse de Picsou, vol. 1, 2016.

34. Chapter 0, "Of Ducks, Dimes and Destinies», Uncle \$crooge ${ }^{\circ} 297$, février 1996 : https:// inducks.org/issue.php?c=us/US\%20\%20297

Le chapitrage n'est pas non plus mentionné dans la publication initiale de l'histoire dans le magazine suédois Kalle Anka \& C:o (« En krona för ditt öde », Kalle Anka \& C:0, n¹995-24, Suède, juin 1995) : https://inducks.org/story.php?c=D+91249\&search=En\%20krona\%20för\%20ditt\%20öde \% $2 \% \mathrm{A0}$ 
Les pages-titres du chapitre dans les différents pays sont également accessibles sur le site d'archives I.N.D.U.C.K.S. : https://inducks.org/story.php?c=D+91249\#scans

35. Don Rosa, Bonus, « Le Rêve de toute une vie », Intégrale Don Rosa, t. 2, Glénat, 2013 [2002].

\section{ABSTRACTS}

This paper aims at studying the different versions of The Life and Times of Scrooge McDuck by Keno Don Rosa, which was initially published in Denmark by Egmont between 1992 and 1994, before being released in the United States and in France a few years later. Recounting its complex editorial history, I analyse the variations associated with the transition from an episodic narrative release to a complete edition arranged in chapters and their pragmatic and symbolic consequences.

Cet article vise à étudier les différentes versions de La Jeunesse de Picsou de Keno Don Rosa, qui a été initialement publiée au Danemark par Egmont entre 1992 et 1994, avant de paraître aux ÉtatsUnis et en France quelques années plus tard. Retraçant son histoire éditoriale complexe, j'analyse les variations induites par le glissement d'une narration épisodique à une édition complète chapitrée, ainsi que leurs conséquences pragmatiques et symboliques.

\section{INDEX}

Mots-clés: chapitre, épisode, feuilleton, comic book, magazine, culture médiatique Keywords: chapter, episode, serial, comic book, magazine, media culture

\section{AUTHOR}

\section{ANAÏS GOUDMAND}

Anaïs Goudmand est assistante à l'École de Français Langue Étrangère de l'Université de Lausanne. Elle a soutenu en 2018 une thèse intitulée « Récits en partage. Expériences de la sérialité narrative en culture médiatique ", dirigée par Raphaël Baroni (Université de Lausanne) et Jean-Marie Schaeffer (EHESS). Elle a écrit plusieurs articles sur l'économie du récit sériel, parmi lesquels " “Oh my God! They’ve killed..!" Le récit sériel entre autonomie et hétéronomie : conséquences du départ non planifié des acteurs sur la production et la réception des séries télévisées » (Télévision, $\left.{ }^{\circ} 7,2016\right)$. Elle a également co-organisé avec Raphaël Baroni le colloque international « Les avatars du chapitre en bande dessinée » dans le cadre du projet ANR «Chapitres - Pratiques et poétiques du chapitre du XIX ${ }^{\mathrm{e}}$ au XXI ${ }^{\mathrm{e}}$ siècle : génétique, rhétorique de la lecture et transmédialité » (Université de Lausanne, 11-12 mai 2017). 